\title{
Frequency Separation Actuation Resonance Cancellation for Vibration Suppression Control of Two-Inertia System Using Double Motors
}

\author{
Kenji Inukai, and Hiroshi Fujimoto \\ The University of Tokyo \\ Kashiwa, Chiba, Japan \\ Email: inukai@hflab.k.u-tokyo.ac.jp, \\ fujimoto@k.u-tokyo.ac.jp
}

\begin{abstract}
Usually, a humanoid robot using motors has low resonances due to the timing belts at each joint, and the low resonances may deteriorate the response performance and positioning precision. In this paper, a joint of humanoid robot which links the motor side to the load side via timing belt is studied. Regarding this system as a two-inertia system, this paper proposes a novel control method which uses multiple motors in both the motor side and the load side for multi-input-single-output (MISO) system. The proposed method achieves vibration suppression and phase stabilization simultaneously. The implementation is relatively simple since there are only two parameters required in the design. Simulations and experimental results also demonstrate the effectiveness of the proposed control system.
\end{abstract}

\section{INTRODUCTION}

Humanoid robots have been the focus of attention because of the low birthrate and longevity. Recently, improvement of kinematical performance is remarkable through the development of technology. However, bandwidth of feedback control can not be improved because low rigidity of the reduction gear of humanoid robot generates resonance. Therefore, it is necessary to model a plant by a two-inertia system, and introduce a resonance suppression control.

In the motion control field for two-inertia system, many resonance suppression control methods which assume that position sensor is installed only on motor side have been proposed [1]-[6]. However, these methods cannot suppress load side position error due to semi-closed system. Recently, high precision positioning device such as machine tools which can measure not only the position of the motor side but also the position of the load side is increasing. In full-closed loop control system, the cascade control is often used. Self Resonance Cancellation Control (SRC) is proposed as a control method using multiple sensors [7]. This method can suppress the resonance peak and widen the bandwidth. However, SRC is not robust against modeling error. Therefore, it cannot apply to humanoid robot because moment of inertia, centrifugal force, coriolis force, gravity, and frictional force depend on posture. The authors' research group proposed Self Resonance Disturbance Observer (SRCDOB) which regards all modeling errors as disturbances, nominalizes a plant, and improves robustness against modeling errors. The robust resonance suppression control for two-inertia system was achieved by combining SRC and SRCDOB [8]. Furthermore, Frequency Separation

\author{
Taro Takahashi \\ Advanced Technology Engineering Department, \\ Partner Robot Division, \\ Toyota Motor Corporation \\ Tokyo, Japan \\ Email: taro_takahashi@mail.toyota.co.jp
}

Self Resonance Cancellation (FS-SRC) was proposed for XYgantry-stage [9]. It achieves Vibration suppression and phase stabilization.

Conventional methods, such as SRCDOB and FS-SRC are robust resonance suppression control methods in singleinput-multi-output (SIMO) systems using an actuator and multiple sensors. As well, a robust resonance suppression control would be achieved in a multi-input system. When the robust resonance suppression in multi-input system is applied in a humanoid robot, miniaturization of joint by distributed placement of motors is possible.

In this paper, in order to achieve a robust resonance suppression control for humanoid robot, a novel feedback system is proposed in a multi-input-single-output (MISO) system using double motors and single angle sensor. The proposed feedback system has two features. First one is that the effect of the vibration suppression. The other is that the phase stabilization of the resonance mode can be tuned by only two parameters. This proposed method is a basic study of multi-input system.

The composition of this paper is shown below. Firstly, the composition of the 3-joint leg robot created for basic examination of control design in a robot leg, and model of twoinertia system are explained. Secondly, the theory of proposed system for two-inertia system using double motors and single angle sensor is stated. Finally, simulations and experiments are performed to show the advantages of the proposed system.

\section{3-JOINT LEG ROBOT AND EXPERIMENT EQUIPMENT TO SIMULATE A JOINT}

\section{A. Basic Composition of Joint of Humanoid Robot}

The authors fabricate 3-joint leg robot shown in Fig. 1 in order to do some basic examination of control design in a robot leg. The 3-joint leg robot has hip, knee, and ankle joints. The joint of load side is connected to a harmonic gear through a timing belt from a motor shown in Fig. 2. Modeling in twoinertia system for each joint is necessary under the influence of the elasticity of each timing belt. Validity of modeling the joint in a two-inertia system has been shown in previous research [8]. 


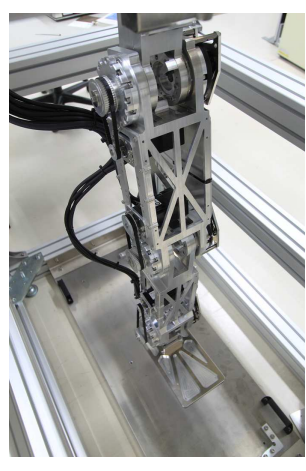

Fig. 1. 3-joint Leg Robot

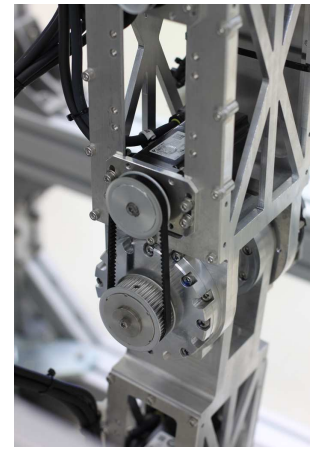

Fig. 2. Composition of Transmission of Joint

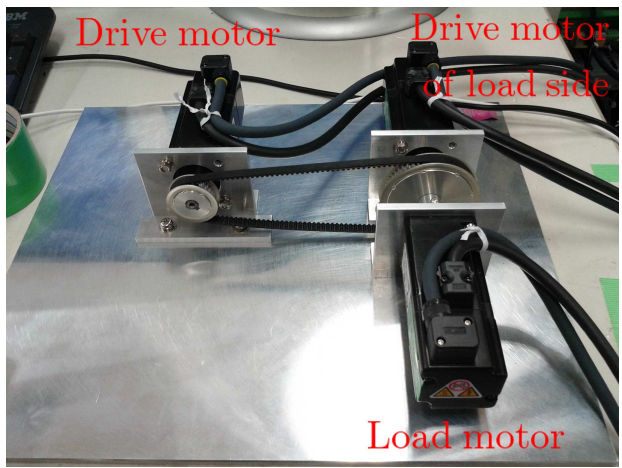

Fig. 3. Experiment equipment to simulate joints of humanoid robot

\section{B. Experiment equipment to simulate a joint of humanoid robot}

The experiment equipment to simulate a humanoid robot joint is shown in Fig. 3. In this equipment, the motor side and the load side are connected through a timing belt, and the drive motor of load side is mounted to apply the proposed control system, and the load motor is mounted to simulate the posture change of the humanoid robot. The drive motor of load side and the load motor is connected by rigid coupling. This experiment equipment is modeled as a two-inertia system shown in Fig. 4. The motor moment of inertia, the motor damping coefficient, the load moment of inertia, the load damping coefficient, and the spring constant are denoted as $J_{M}, B_{M}, J_{L}, B_{L}$, and $K$ respectively. The gear ratio is denoted as $r$. The motor side angle is denoted as $\theta_{M}$, the load side angle is denoted as $\theta_{L}$, the motor torque is denoted as $T_{M}$, and the load side torque is denoted as $T_{L}$. The block diagram of two-inertia system is shown in Fig. 5. For a two-inertia system, transfer functions from motor torque $T_{M}$ to motor angle $\theta_{M}$ and load angle $\theta_{L}$, and from load side torque $T_{L}$ to motor angle $\theta_{M}$ and load angle $\theta_{L}$ represent by

$$
\begin{aligned}
P_{M M}(s)=\frac{\theta_{M}}{T_{M}} & =\frac{J_{L} s^{2}+B_{L} s+K r^{2}}{D}, \\
P_{L M}(s)=\frac{\theta_{L}}{T_{M}} & =\frac{K r}{D}, \\
P_{M L}(s)=\frac{\theta_{M}}{T_{L}} & =\frac{K r}{D}, \\
P_{L L}(s)=\frac{\theta_{L}}{T_{L}} & =\frac{J_{M} s^{2}+B_{M} s+K}{D},
\end{aligned}
$$

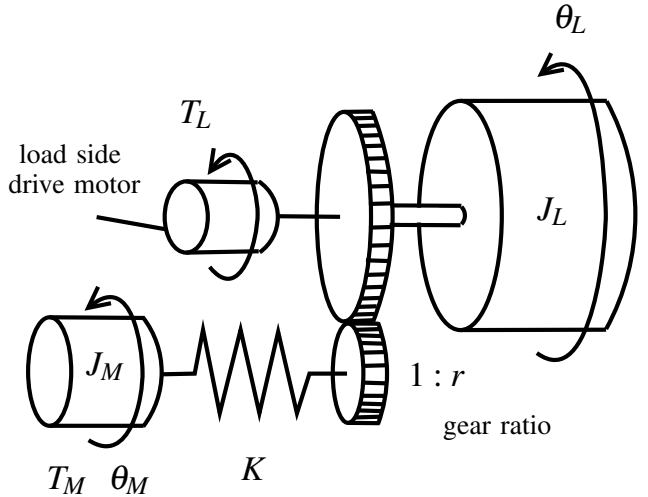

Fig. 4. Model of two-inertia system

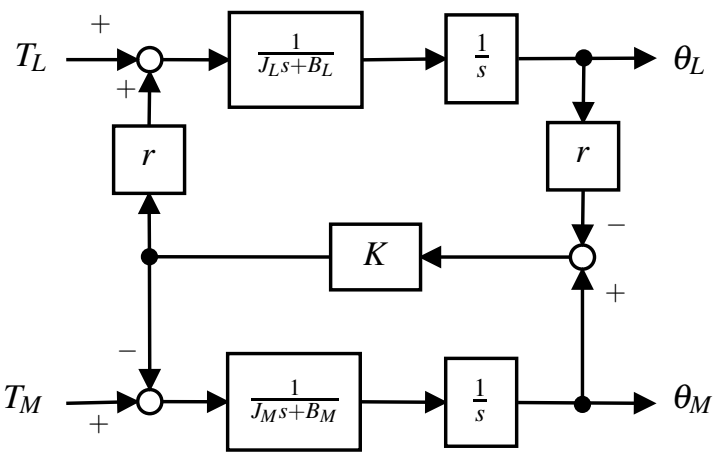

Fig. 5. Block diagram of Two-inertia system

where

$$
\begin{aligned}
D= & J_{M} J_{L} s^{4}+\left(J_{M} B_{L}+J_{L} B_{M}\right) s^{3} \\
& +\left(r^{2} J_{M} K+J_{L} K+B_{M} B_{L}\right) s^{2} \\
& +\left(r^{2} B_{M} K+B_{L} K\right) s .
\end{aligned}
$$

From the denominator polynomial of (1)-(4) and the numerator polynomial of (1), the resonant angular frequency and the antiresonant angular frequency given by

$$
\begin{aligned}
& \omega_{p}=\sqrt{K\left(\frac{r^{2}}{J_{L}}+\frac{1}{J_{M}}\right)}, \\
& \omega_{z}=\sqrt{\frac{K r^{2}}{J_{L}}} .
\end{aligned}
$$

The bode diagrams of the frequency response characteristic from motor torque reference $T_{M}$ to motor angular velocity $\dot{\theta}_{M}$ and two-inertia system fitting model are shown in Fig. 6. The resonance at $55 \mathrm{~Hz}$ comes from the timing belt. Nominal values are decided by this frequency response characteristic are shown in Table. I. This model is not exact in the low frequency region due to nonlinear friction. In this paper, this experiment equipment is examined.

\section{FREQUENCY SEPARATION ACTUATION RESONANCE CANCELLATION CONTROL}

\section{A. Actuation Resonance Cancellation Control}

This section describes about the proposed method called Actuation Resonance Cancellation Control (ARC). Conven- 

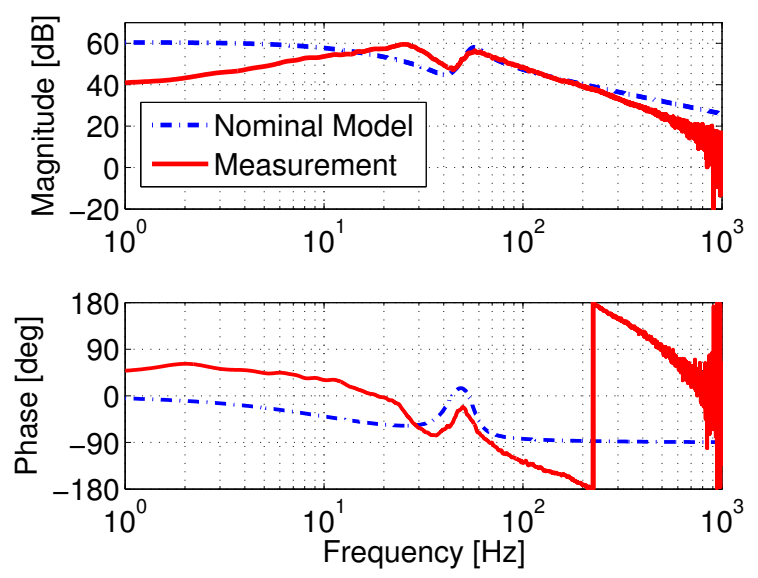

Fig. 6. Frequency characteristic of Experiment equipment

TABLE I. PARAMETER OF EXPERIMENT EQUIPMENT

\begin{tabular}{llr}
\hline \hline$J_{M}$ & $\mathrm{~kg} \cdot \mathrm{m}^{2}$ & $8.0 \times 10^{-6}$ \\
$B_{M}$ & $\mathrm{~N} \cdot \mathrm{m} /(\mathrm{rad} / \mathrm{s})$ & $5.0 \times 10^{-4}$ \\
$J_{L}$ & $\mathrm{~kg} \cdot \mathrm{m}^{2}$ & $2.2 \times 10^{-5}$ \\
$B_{L}$ & $\mathrm{~N} \cdot \mathrm{m} /(\mathrm{rad} / \mathrm{s})$ & $1.8 \times 10^{-3}$ \\
$K$ & $\mathrm{~N} \cdot \mathrm{m} /(\mathrm{rad})$ & 0.30 \\
$r$ & & 2.0 \\
\hline
\end{tabular}

tional resonance cancellation control such as SRC cancels the resonance by using encoders attached at both the motor side and the load side and using single motor. The proposed system cancels the resonance of the plant by using motors attached at both the motor side and the load side and using single encoder attached at load side. The block diagram of ARC is shown in Fig. 7. In this system, transfer functions from input torque $T_{i n}$ to load angle $\theta_{L}$, from input torque $T_{i n}$ to motor angle $\theta_{M}$ are defined as

$$
\begin{gathered}
P_{L}(s)=\frac{\theta_{L}}{T_{i n}}=\left(\alpha+\frac{\beta}{s}\right) P_{L M}(s)+\left(\gamma+\frac{\delta}{s}\right) P_{L L}(s),(8) \\
P_{M}(s)=\frac{\theta_{M}}{T_{i n}}=\left(\alpha+\frac{\beta}{s}\right) P_{M M}(s)+\left(\gamma+\frac{\delta}{s}\right) P_{M L}(s) .(9)
\end{gathered}
$$

The controller gains $\alpha, \beta, \gamma$, and $\delta$ are determined to cancel the resonance of the plant. Although there are an infinite number of solutions, these parameters are determined as follows:

$$
\begin{aligned}
\alpha_{A R C} & =\frac{J_{M}}{J_{M}+\frac{J_{L}}{r^{2}}}, \\
\beta_{A R C} & =\frac{B_{M}}{J_{M}+\frac{J_{L}}{r^{2}}}, \\
\gamma_{A R C} & =\frac{J_{L}}{r\left(J_{M}+\frac{J_{L}}{r^{2}}\right)}, \\
\delta_{A R C} & =\frac{B_{L}}{r\left(J_{M}+\frac{J_{L}}{r^{2}}\right)} .
\end{aligned}
$$

The parameter $\alpha_{A R C}$ means inertia ratio. Then, in the case of the above parameters, the transfer functions $P_{M}(s)$ and $P_{L}(s)$

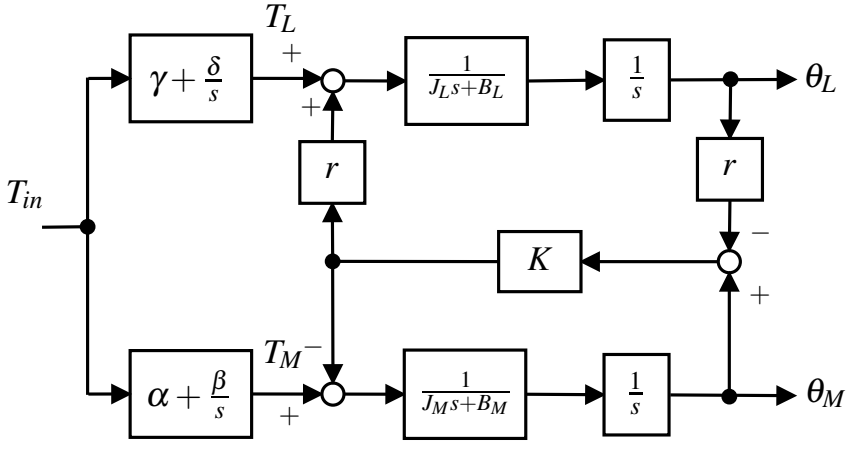

Fig. 7. Block diagram of the ARC

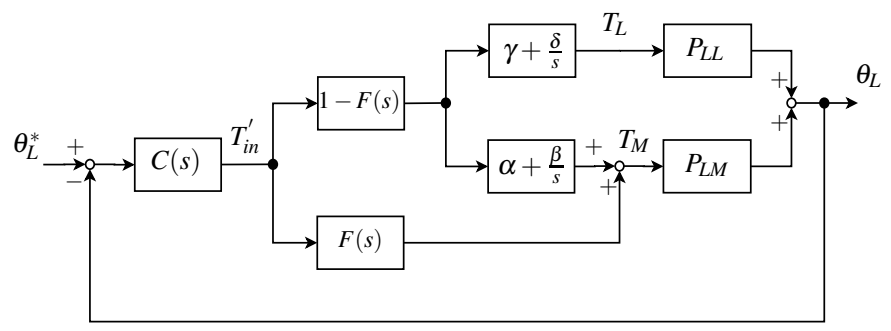

Fig. 8. Block diagram of the FS-ARC system

are represented by

$$
\begin{gathered}
P_{L, A R C}(s)=\frac{\theta_{L}}{T_{i n}}=\frac{1}{r\left(J_{M}+\frac{J_{L}}{r^{2}}\right) s^{2}}, \\
P_{M, A R C}(s)=\frac{\theta_{M}}{T_{i n}}=\frac{1}{\left(J_{M}+\frac{J_{L}}{r^{2}}\right) s^{2}} .
\end{gathered}
$$

As shown in (14) and (15), the resonance of the plant is canceled.

This proposed method is the same as direct driving the load side. This proposed system is applicable in the case of the drive motor of load side which is enough to direct drive. This control system is robust against spring constant $K$ because controller gains do not include $K$.

\section{B. Frequency Separation Actuation Resonance Cancellation Control}

In this section, the Frequency Separation Actuation Resonance Cancellation Control (FS-ARC) is proposed. This proposed method is applicable in the case of drive motor of load side which is not enough to direct drive. The block diagram of FS-ARC is shown in Fig. 8. The transfer function from $T_{i n}^{\prime}$ to the load angle $\theta_{L}$ is defined as

$$
P_{F S-A R C}(s)=\frac{\theta_{L}}{T_{i n}^{\prime}}=F(s) P_{L M}(s)+\{1-F(s)\} P_{L}(s),
$$

where $F(s)$ is the first order low pass filter (LPF) as follows:

$$
F(s)=\frac{2 \pi f_{L P F}}{s+2 \pi f_{L P F}} .
$$

ARC method cancels the resonance by making the unobservable of the resonant mode from reference angle to load 

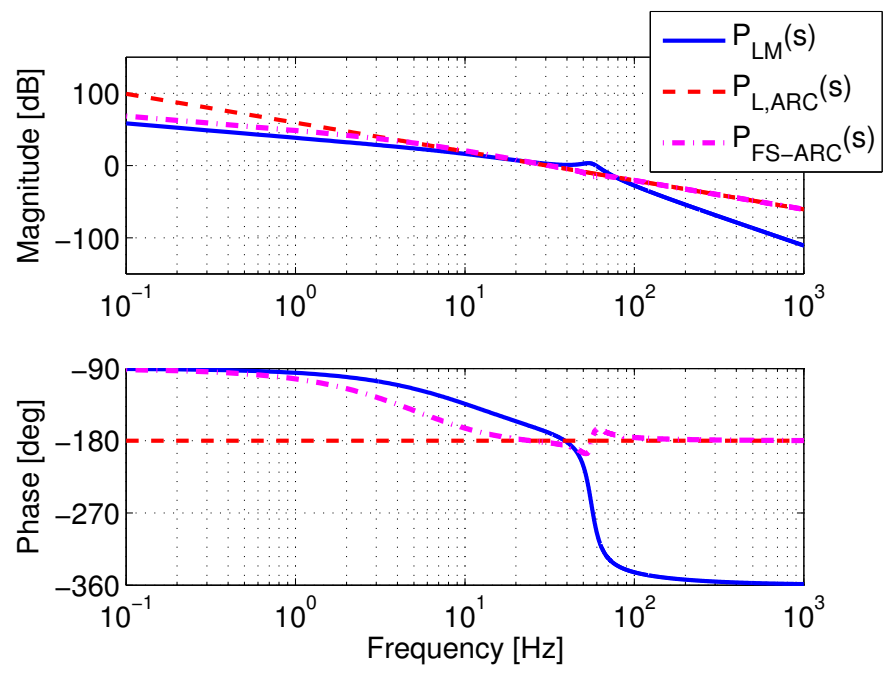

Fig. 9. Frequency characteristic of Frequency Separation Actuation Resonance Cancellation Control

angle. Therefore, unexpected vibration occurs by disturbance. On the other hand, FS-ARC dose not cancel the resonance mode completely. However, FS-ARC method can suppress vibration due to disturbance. FS-ARC method can adjust the suppression performance of reference and disturbance. In FSSRC method, $\alpha$ of $P_{L}(s)$ is variable in the range from 0 to 1 . $\beta, \gamma$ and $\delta$ is expressed using $\alpha$ as follows:

$$
\begin{aligned}
\beta & =\frac{B_{M}}{J_{M}} \alpha, \\
\gamma & =r(1-\alpha), \\
\delta & =\frac{B_{L}}{J_{L}} r(1-\alpha) .
\end{aligned}
$$

The FS-ARC method uses drive motor torque to control low frequency region in which the resonance does not affect, and uses drive motor and drive motor of load side in high frequency region where the resonance exists. FS-ARC method includes ARC method. FS-ARC is equivalent to ARC in the case of $\alpha=\alpha_{A R C}$ and $f_{L P F}=0 \mathrm{~Hz}$.

The bode diagram of the $P_{L M}(S), P_{L, A R C}(S)$ and $P_{F S-A R C}(S)$ shown in Fig. 9. The solid line is frequency characteristic of $P_{L M}(S)$, the broken line is frequency characteristic of $P_{L, A R C}(S)$, and the dot-dashed line is frequency characteristic of $P_{F S-A R C}(S)$ at $\alpha=\alpha_{A R C}$. The cut-off frequency of the LPF of FS-ARC is $5 \mathrm{~Hz}$, because the resonant frequency of the plant is $55 \mathrm{~Hz}$.

The resonance mode of plant is canceled by applying resonance cancellation. The resonance mode of plant is almost canceled by applying FS-ARC. In addition, the band width of feedback system can be improved because the phase delay of $P_{F S-A R C}(s)$ at high frequency region is $-180^{\circ}$.

Next, the stability of the proposed method is analyzed. The feedback controller is designed for $P_{L, A R C}(s)$ which is the rigid mode of load side. In this paper, the feedback controller is designed as a PID controller. The parameters of the controller is determined based on the pole placement design to poles place at $20 \mathrm{~Hz}$. The parameters are determined simply because the feedback controller of this system designed for rigid plant.

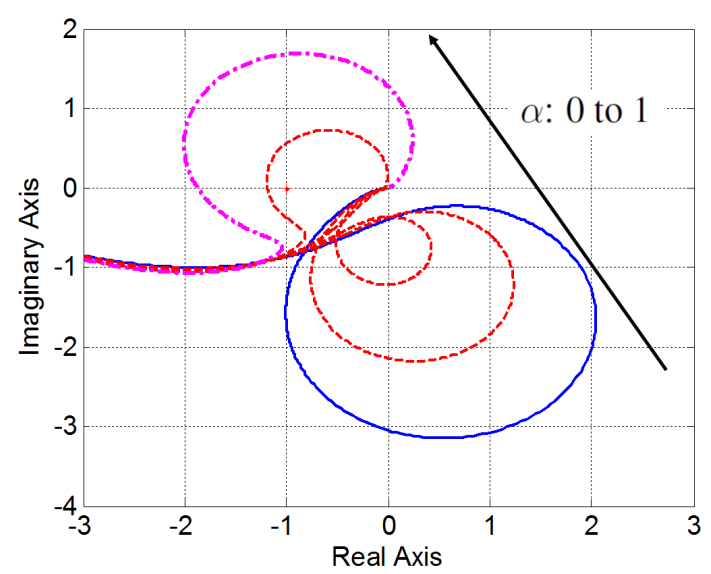

Fig. 10. Nyquist diagram of the proposed system when $\alpha$ is variable in the range from 0 to 1 and $f_{L P F}=0 \mathrm{~Hz}$

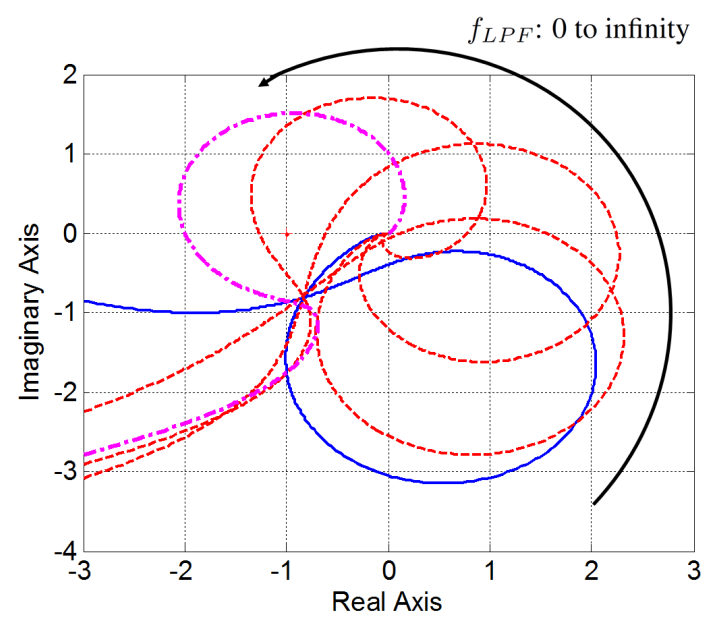

Fig. 11. Nyquist diagram of the proposed system when $f_{L P F}$ is variable in the range from 0 to infinity and $\alpha=0$

Fig. 10 shows the nyquist diagram of FS-ARC system when $\alpha$ varies from 0 to 1 and $f_{L P F}$ is $0 \mathrm{~Hz}$. Fig. 11 shows the nyquist diagram of FS-ARC system when $f_{L P F}$ is changed from 0 to infinity and $\alpha$ is 0 . The solid line in Fig. 10 and Fig. 11 are the case that $\alpha$ is 0 and $f_{L P F}$ is 0 . (i.e. The resonance mode of $P_{F S-A R C}(s)$ equals to that of $P_{L L}(s)$.) The dot line in Fig. 10 is the case that $\alpha$ is 1 and $f_{L P F}$ is 0 . (i.e. The resonance mode of $P_{F S-A R C}(s)$ equals to that of $P_{L M}(s)$.) The dot-dashed line in Fig. 11 is the case that $\alpha$ is 0 and $f_{L P F}$ is infinity. (i.e. The resonance mode of $P_{F S-A R C}(s)$ equals to that of $P_{L M}(s)$.)

Here, it is noticed that both the amplitude and the direction of the circle of the resonance mode can be designed between the own characteristics $P_{L L}(s)$ and $P_{L M}(s)$ in the nyquist diagram. In other words, the vibration suppression and the phase stabilization of the resonance mode can be tuned simultaneously only by the two parameters $\alpha$ and $f_{L P F}$. Because of this feature, FS-ARC is the dual control system of FS-SRC which is applied for SIMO system [9].

The tuning of the two parameters $\alpha$ and $f_{L P F}$ is determined simply. First the vibration suppression is determined by the 


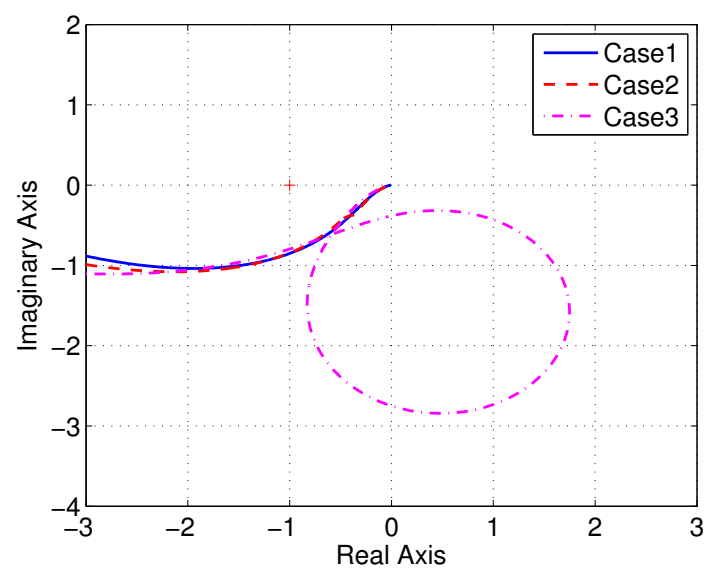

Fig. 12. Nyquist diagram in the simulation

tuning of $\alpha$. Second the tuning of $f_{L P F}$ makes biggest the phase margin of the closed loop. For example, the circle of the resonance mode is located far from the point $(-1,0)$ in the nyquist diagram as much as possible.

\section{Simulations}

Frequency responses of ARC and FS-ARC are evaluated in a simulation. The simulation is performed in the three cases as follows:

- $\quad$ Case 1: ARC $\left(\alpha=\alpha_{A R C}, f_{L P F}=0 \mathrm{~Hz}\right)$, Poles of $\left(1+P_{L, A R C}(s) C(s)\right)^{-1}: 30 \mathrm{~Hz}$,

- $\quad$ Case 2: $\alpha=\alpha_{A R C}, f_{L P F}=1.0 \mathrm{~Hz}$, Poles of $\left(1+P_{L, A R C}(s) C(s)\right)^{-1}: 25 \mathrm{~Hz}$,

- $\quad$ Case 3: $\alpha=0.1, f_{L P F}=5.0 \mathrm{~Hz}$, Poles of $\left(1+P_{L, A R C}(s) C(s)\right)^{-1}: 32 \mathrm{~Hz}$.

The controller is redesigned to equalize the phase margins of Case 2 and Case 3 to that of Case 1. Phase margin is $43.5 \mathrm{deg}$. The nyquist diagram of three cases is shown in Fig. 12. The resonance mode is almost canceled in Case 1 and Case 2. The circle of the resonance mode is located far from the point $(-1$, 0 ) in Case 3. The phase stabilization of the resonance mode is tuned.

\section{EXPERIMENT}

Frequency responses and time responses of ARC and FSARC are evaluated in experiments with experiment equipment shown in Fig. 3. The experiment is performed in the three cases as same as the simulation.

\section{A. Freqency Responses}

The nyquist diagram of Frequency Separation Actuation Resonance Cancellation Control is evaluated in experiment . The experiment result is shown in Fig. 13. The resonance mode is almost canceled in Case 1 and Case 2. The direction of the resonance mode could be located far from the point $(-1,0)$ in Case 3.

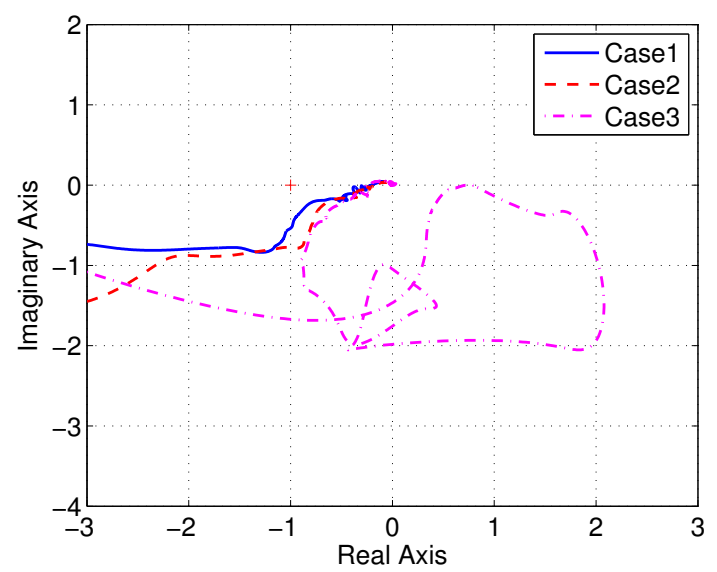

Fig. 13. Nyquist diagram in the experiment

\section{B. Time Responses}

Time responses of the ARC and FS-ARC systems are evaluated by step response and load side disturbance addition experiment of experimental equipment as shown Fig.3

1) Step Responses: Fig.14 shows the step response of load side angle. Step reference input is added at $0.1 \mathrm{~s}$. In Case 1 , the vibration of the resonance is suppressed. In Case 2, the vibration of the resonance is suppressed. However, the overshoot is increased from Case 1. In Case 3, the overshoot is decreased, but the time response is vibrationally because controller parameter $\alpha$ is not $\alpha_{A R C}$.

Fig. 15 shows the torque of load side drive motor in Case 1 and Case 2. By comparing the Case 1 and Case 2, peak torque of Case 2 is smaller than that of Case 1. The drive motor of load side can be smaller $25 \%$ using FS-ARC system at $f_{L P F}=1$ $\mathrm{Hz}$.

2) Load Side Disturbance Response: In this section, the ARC and FS-ARC system is evaluated in load Side disturbance torque addition experiment. The step torque is added to load motor. The load angle reference is set to 0 , and step disturbance torque $0.02 \mathrm{~N} \cdot \mathrm{m}$ is added at time $0.1 \mathrm{~s}$. The experimental result is shown in Fig.16. The time responses of Case 1 and Case 2 is vibrating at primary resonance frequency. In Case 3, the disturbance suppression efficiency is high and the vibration is suppressed.

Case 1 attaches weight to the suppression performance in step reference response. Case 2 attaches weight to the miniaturization of load side drive motor. Case 3 attaches weight to the suppression performance in load side disturbance suppression.

\section{CONCLUSION}

Resonance cancellation in MISO system which is attached motor at both the motor side and the load side is proposed. ARC and FS-ARC can be applied for two-inertia system such as a joint of humanoid robot. In simulation, the resonance mode is canceled, and the vibration suppression and the phase stabilization of the resonance mode can be tuned simultaneously only by the two parameters $\alpha$ and $f_{L P F}$. The 


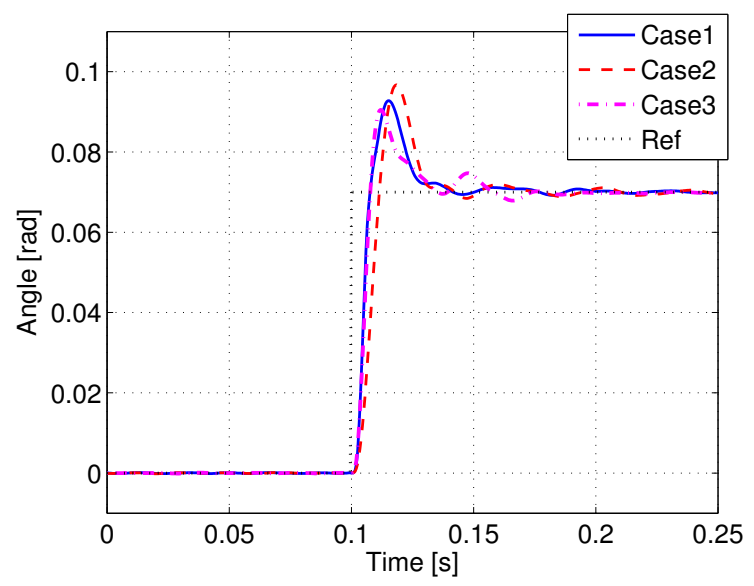

Fig. 14. Experimental results of step responses

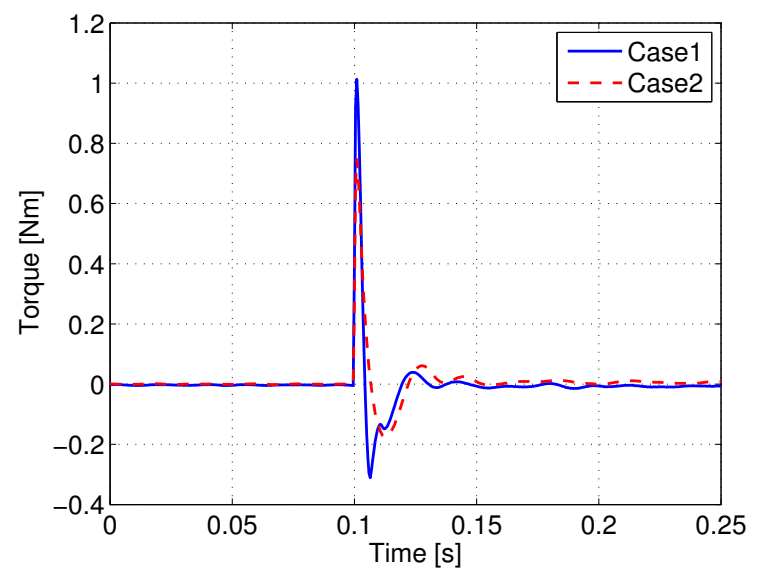

Fig. 15. Torque of load side drive motor of step responses

experimental results show that vibration suppression efficiency of step reference response and disturbance response are tuned by $\alpha$ and $f_{L P F}$. The load side drive motor for vibration suppression can be smaller using FS-ARC in comparison to ARC.

Conventional resonance cancellation control methods, such as SRC and SRCDOB in SIMO system control the virtual angle which is canceled resonance. On the other hand, the proposed system in MISO system controls the load side angle $\theta_{L}$.

\section{REFERENCES}

[1] K. Yuki, T. Murakami, and K. Ohnishi, "Vibration Control of a 2 Mass Resonant System by the Resonance Ratio Control", Trans. Inst. Elect. Eng. Jpn. vol. 113-D, no. 10, pp. 1162-1169, 1993.

[2] Y. Hori, H. Sawada, and Y. Chun, "Slow resonance ratio control for vibration suppression and disturbance rejection in torsional system", IEEE Trans. Ind. Electron., vol. 46, no. 1, pp. 162-168, 1999.

[3] Y. Hori, H. Iseki, and K. Sugiura, "Basic consideration of vibration suppression and disturbance rejection control of multi-inertia system using SFLAC (state feedback and load acceleration control)," IEEE Trans. Ind. Appl., vol. 30, no. 4, pp. 889-896, 1994.

[4] K. Ohishi, N. Shimada, and T. Miyazaki, "High-performance robot motion control based on zero-phase notch filter for industrial robot", The 11th IEEE International Workshop on Advanced Motion Control, pp.626-630, 2010.

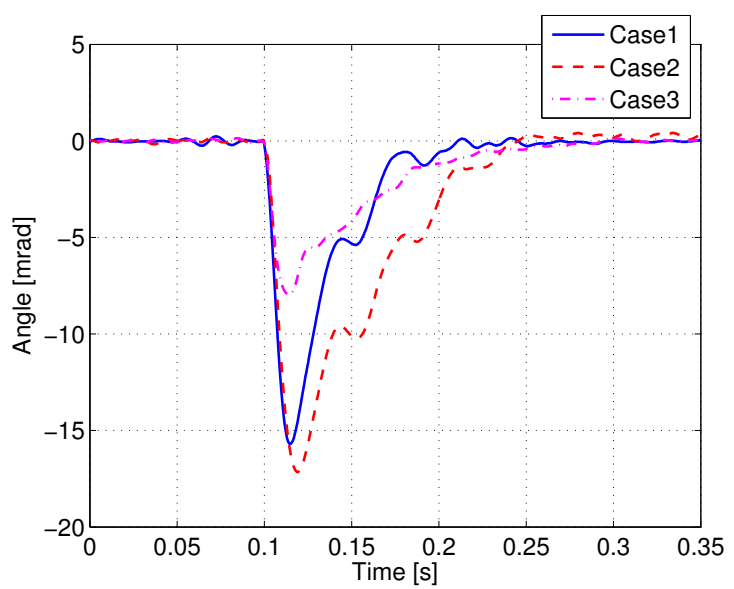

Fig. 16. Experimental results of load side disturbance responses

[5] G. Zhang, J. Furusho, "Speed Control of Two-Inertia System by PI/PID Control”, IEEE Trans. Ind. Electron., vol. 47, no. 3, pp. 603-609, 2000.

[6] Y. Marushita, H. Ikeda, and H. Sugie, "Vibration Suppression Control using the Load-side Acceleration Feedback," in Proc. the 33th Annual Conference of IEEE Industrial Electronics Society, pp. 810-815, 2007.

[7] Y. Seki, H. Fujimoto, and K. Saiki, "Optimal Mechanical Parameter Design Using Self Resonance Cancellation Control for Gantry-Type High Precision Stage", in Proc. The 12th International Workshop on Advanced Motion Control, 2012.

[8] M. Aoki, H. Fujimoto, Y. Hori, and T. Takahashi, "Robust Resonance Suppression Control based on Self Resonance Cancellation Disturbance Observer and Application to Humanoid Robot", in Proc. IEEE International Conference on Mechatronics, pp. 623-628, 2013.

[9] K. Sakata, H. Asaumi, K. Hirachi, K. Saiki, and H. Fujimoto, "Frequency Separation Self Resonance Cancellation for Vibration Suppression Control of a Large-Scale Stage Using Multiple Position Sensors", in Proc. the 39th Annual Conference of IEEE Industrial Electronics Society, pp.64786483, 2013. 